\title{
LES PARADOXES DE LA TOTALITÉ OU LES « MILLE PARTIES » DU LANGAGE
}

\author{
Sylvain Auroux \\ UMR 7597 « Histoire des théories linguistiques »
}

\section{Résumé}

Les paradoxes de la totalité concernent les langues et leurs parties (dialectes; mots ; propositions). Nous montrerons que ce que l'on entend par «langue» (comme dans « langue nationale ») est de l'ordre, d'un côté, de l'imaginaire, de l'autre, de la construction sociale et technique.

\section{Mots-clés}

Tout, parties, mots, propositions, définition, langues, langage, discours, acte de parole, hyperlangue

\section{Abstract}

The part-whole relationship pertains to languages and their components (dialects, words, clauses). We shall argue that what we call "language" (as in "national language") is both an imaginary concept and a social and technical construct.

\section{Keywords}

Whole and parts, words, clauses, definition, languages, language, speech, speech act, hyperlanguage

Il existe des concepts embarrassants, dont le statut est loin d'être clair et dont l'exploration conduit à des paradoxes (assertions qui ne correspondent pas à ce à quoi on s'attendait au départ), voire à des contradictions. Les plus importants sont bien connus ; il s'agit de l'infini, qui a posé de sérieux problèmes aux mathématiques, et de l'universalité, qui reste encore la croix des sciences du langage. Kant a vu l'origine des problèmes posés par ce type de concept dans l'application empirique illégitime du fonctionnement de l'entendement. La totalité a d'abord posé des problèmes avec le concept de « vivant ». Si les sciences du langage connaissent, dès leur départ en Grèce, la problématique «tout/parties » (les «parties du discours »), celle-ci n'est devenue embarrassante qu'avec la reformulation du concept de langue due aux romantiques allemands (par exemple, Humboldt et l'idée qu'une langue apparaît tout d'un coup). Un nouveau statut de la totalité a commencé à être pensé avec le structuralisme (particulièrement européen), qui conduira Lévi-Strauss (1950) à défendre l'idée d'un surgissement 
instantané de l'ordre symbolique. Pour en comprendre les enjeux, il faut se référer à l'axiomatique de la totalité qui figure dans la doxa, ce que l'on peut appeler la définition de la « totalité ordinaire » (TO).

\section{LES AXIOMES DE LA TOTALITÉ}

Une totalité suppose : a) Une unité (un, le) ; b) Une pluralité (parties, éléments). La conception d'une totalité et du rapport à ses parties parait quelque chose de relativement simple :

Ax. 1 : Le tout est la somme des parties (on a le tout dès que l'on a les parties, les parties précédent le tout) ;

Cor. 1-i : On passe des parties au tout par sommation ;

Cor. 1-ii : L'ajout d'une partie change le tout, mais ne change pas les autres parties ;

Ax. 2 : Le tout est plus grand que la partie ;

Ax. 3 : Une partie est un élément ;

Ax. 4 : On passe du tout à la partie par division ;

Cor. 4-i : La division est l'opération inverse de la sommation (compositionnalité).

Bien entendu cette simplicité n'est qu'apparente. Elle dissimule déjà quelques questions dont la réponse n'est pas évidente :

a) Qu'est-ce qui fait qu'un tout est un tout ? On admettra difficilement que les pipes que j'ai devant moi dans le cendrier forment une totalité. Mais je puis employer l'expression « toutes mes pipes » et ce n'est qu'un hasard si l'expression convient à celles qui sont dans mon cendrier. Cela est parfaitement clair dans le cas d'une collection d'objets finis ; cela devient obscur si l'on envisage l'infini. Que désigne l'expression : «Toutes les phrases de la langue française $»$ ?

b) Qu'est-ce qui fait qu'un élément (un « étranger ») n'appartient pas à un tout ? On n'hésite pas à dire « ceci n'est pas français ». Déjà les grammairiens romains se posaient la question de savoir si la " latinité » d'une expression pouvait provenir d'une autorité assignée, comme celle de l'empereur.

c) Faut-il qu'il y ait un extérieur pour qu'il y ait un tout ? Pour la cosmologie antique, il n'y a rien en dehors du cosmos fini. Si une flèche se fichait sur la « frontière », ce qui en dépasserait n'existerait pas. Au XVIII ${ }^{\mathrm{e}}$ siècle on parlait volontiers de la langue primitive. Qu'elle fût unique correspondait, chez un Court de Gébelin (Le Monde Primitif), à une idée archaïque du cosmos et à l'unité de l'espèce humaine. On sait que la condition épistémologique de développement de la grammaire comparée a été de bloquer par l'arbitraire 
linguistique la possibilité de remonter de la diversité des langues actuelles à leur origine potentielle (Auroux 2007). Pour la logique mathématique, une théorie ne peut être consistante que si au moins l'une des expressions bien formées $(E d f)$ n'est pas une thèse du système (non- $p$ et $p$ sont des propositions bien formées et l'une doit être exclue pour éviter la contradiction).

d) Que serait le tout de tous les touts ? La cosmologie antique pose la réalité finie du cosmos, mais la physique moderne entraina Kant à faire de la question cosmologique la première antinomie de la Raison pure. Pour la résoudre, on doit admettre que l'idée de la totalité de l'Univers est une simple idée régulatrice sans contenu empirique. Certes nous admettons aujourd'hui que le big bang est l'origine de l'univers. Cela signifie simplement qu'il n'y a pas de sens empirique à parler d'un avant ou d'un dehors, puisque espace et temps se définissent dans l'évolution même de l'univers. Mais le concept d'une infinité de big bangs ne semble pas contradictoire, simplement dénué de toute possibilité de contenu empirique. Les mathématiques ont longtemps buté sur la question du nombre de tous les nombres; ce n'est qu'au XIX siècle qu'on admit que l'infini est un nombre ou, plutôt, qu'il se fragmente en une classe de nouveaux nombres, les transfinis de Cantor. Si l'on parle de l'ensemble de tous les ensembles, il faut admettre qu'il fait partie de lui-même et qu'il ne fait pas partie de lui-même. B. Russell, pour éviter les paradoxes, dut admettre que l'ensemble de tous les ensembles n'était pas un ensemble. La totalité des langues n'est certainement pas une langue universelle.

Les sciences du langage n'ont pas manqué d'un rapport fort avec TO. Elles lui ont parfois donné un modèle susceptible de s'étendre au monde physique : on sait que l'atomisme usait volontiers de l'exemple de l'alphabet, conçu comme un ensemble fini d'éléments discontinus permettant d'engendrer des composés ad libitum. De fait, on disposait également de modèles «naturels »:

(i) La plage, le tas de sable vs les grains de sable (individus, atomes);

(ii) La mer, le bol d'eau, la flaque, la goutte vs l'écoulement de l'eau ;

(iii) Un corps vivant vs une tête, un bras, une jambe, etc.

Ces trois modèles, permettent de faire des distinctions. (i) et (ii) ont des parties de même « nature » par opposition à (iii), ce qui justifie l'opposition aristotélicienne entre les totalités "homéomères » et "an-homéoméres ». (iii) présente une particularité : un tas de membres épars n'est pas un corps vivant, le tout (le corps) n'est pas l'ensemble des parties (les membres), telles qu'elles existent dans leur séparation, voire même dans leur réunion amorphe (le cadavre) : il faut quelque chose de plus (Aristote : "L'âme est la forme d'un corps en puissance de vie "). Dans le cas de (ii), on ne parvient pas à atteindre clairement l'élément, ni à sommer ce qui correspond au niveau n-1 pour aboutir au niveau n. Cette sommation n'est pas au reste assurée pour le modèle (i) : à partir de combien de grains de sable obtient-on un tas ? La divisibilité (ou plutôt la séparabilité) n'est assurée que pour les petites quantités, c'est-à-dire pas la plage, mais le sable dans le sablier qui 
mesure le temps. Pour les homéomères du modèle (ii) on peut faire des remarques analogues (la clepsydre utilise l'eau comme le sablier, le sable, et lui est bien antérieure). Dans les deux cas l'élément n'est pas une donnée « naturelle », il y a de gros et de petits grains de sable, comme il y a de grosses et de petites gouttes d'eau. Un grain ou une goutte est le grain ou la goutte de quelque chose. Le sable ou l'eau sont des " masses » qui doivent être données avant leurs « éléments ». Pourtant ce ne sont pas des « touts », chacun constitue la substance avec quoi on peut faire un tout (la plage, la mer).

Qu'un tout soit fini et divisible en parties n'implique pas qu'il soit décomposable en éléments ultimes auto-subsistants. On connaît les paradoxes de Zénon sur l'espace. Entre le départ de la flèche et la cible, la distance est bien finie, mais la flèche doit passer par la moitié de cette distance et la moitié de cette moitié, et ainsi de suite à l'infini. La solution d'Aristote consiste à distinguer a) le niveau de la totalité (l'acte), celui de la trajectoire et b) le niveau de la partie (la puissance), celui des différents points de la trajectoire. Ceux-ci ne sont pas en acte dans la trajectoire, au sens propre, ils n'en sont pas des parties.

Des siècles de réflexion ont mis en cause la simplicité de TO. Nous disposons de résultats clairs qui la remettent en cause comme modèle universel.

L'Ax. 1 et son Cor. 1 semblent incompatibles avec la divisibilité à l'infini ; dans ce cas, le plus simple est d'admettre que le tout précède les parties. On pourrait voir la première formulation de cette idée chez Aristote, si pour le stagirite l'être en puissance n'était pas une forme d'existence. Kant est plus clair : l'espace et le temps ne sont pas des réalités, ce sont des formes de notre perception de la réalité, elles sont données avant. Les mathématiciens admettent que dans tout segment de droite, il y a une infinité de points (comme il y en a dans toute droite infinie), mais ils définissent le point comme l'intersection de deux droites, pas comme un " élément de la droite ». Avec l'apparition du calcul différentiel on s'interrogea sur l'existence des infiniment petits (dx), que Leibniz admettait, mais que les mathématiciens contournèrent longtemps avec la notion de limite. Ils ne réapparaitront qu'avec la naissance, au $\mathrm{XX}^{\mathrm{e}}$ siècle, de l'analyse non-standard.

Dès le XVII ${ }^{e}$ siècle, on sait (Galilée, Leibniz) que l'Ax. 2 ne fonctionne pas non plus. Une suite infinie peut avoir une somme finie. La partie peut être égale au tout : l'ensemble des nombres pairs est une partie de l'ensemble des entiers naturels, mais on peut les mettre en correspondance un à un (on numérote les nombres pairs). Aujourd'hui, on a une infinité d'infinis (les transfinis de Cantor). L'égalité tout/partie est la propriété même de l'infini.

L'axiome 3, qui identifie partie et élément, n'est pas non plus accepté par la théorie des ensembles. Selon un célèbre théorème de Cantor, le cardinal de l'ensemble des parties d'un ensemble est supérieur au cardinal de cet ensemble (le nombre de ses éléments). Tout le monde n'a pas accepté la théorie des ensembles et son idée de base selon laquelle pour un $\mathrm{x}$ avoir la propriété $\mathrm{p}$ est identique au fait pour x d'appartenir à l'ensemble $\mathrm{P}$ (Russell). Ce qui suppose une conception 
distributive de la totalité (la propriété p est pareillement « distribuée » dans tous les individus ou si l'on veut, ils partagent cette propriété). La méréologie de Lesniewski admet des touts composés de « morceaux » (l'anse fait partie de la tasse). La conception ensembliste suppose également la discontinuité : ou x est p ou x n'est pas p. Refuser cette discontinuité revient à accepter des « ensembles flous » (fuzzi sets : on peut être plus ou moins p), dont logiciens et mathématiciens ont constitué la théorie.

Un pas important dans la révision de TO est franchi lorsque l'on admet l'existence de propriétés $\mathrm{p}^{t}$ qui ne peuvent être assertées que de la totalité et en aucun cas de ses parties ou éléments. Il ne s'agit pas de simples propriétés plus ou moins génériques susceptibles de ne pas s'appliquer à tous les individus de la classe concernée (comme c'est le cas dans L'homme est lâche, puisqu'il existe des hommes courageux). La physique nous donne des exemples simples des propriétés $\mathrm{p}^{\mathrm{t}}$ : le débit d'un cours d'eau, la pression d'un gaz, la vie d'un organisme. C'est parce qu'il y a des faits ou propriétés $\mathrm{p}^{t}$ que la cosmologie moderne peut dépasser les antinomies kantiennes et considérer le monde (ou l'univers) comme une totalité réelle : c'est un fait «L'univers est en expansion » et cette expansion on peut la mesurer.

Le vivant est un domaine plus complexe. Au XIX ${ }^{\mathrm{e}}$ siècle on considérait le «fait» de totalité comme une entité à part, la Vie (vitalisme); dans le fond le vitalisme est la poursuite de l'idée antique d'une âme «vivifiant » le corps « inanimé ». Les philosophes (Kant) ont tenté de résoudre la question avec l'idée de « finalité interne » (les organes existent en fonction du tout). Celle-ci sera résorbée dans l'idée plus claire de système autorégulé.

Les sciences humaines ont rencontré les problèmes posés par les «propriétés de totalité » à partir du moment où leur point de départ a été l'individu (cela correspond au Xe siècle à ce que Macpherson ([1962] 1971) qualifie de théories de «l'individualisme possessif »). Le problème principal est celui de passer des représentations des individus à celle du groupe. Il y a incontestablement des volontés individuelles; ou la volonté générale est une fiction ou l'on dispose de protocoles réels pour agréger des volontés individuelles. Le vote démocratique a été proposé comme solution, malgré les paradoxes de Condorcet et, au $\mathrm{XX}^{\mathrm{e}}$ siècle, le théorème d'impossibilité d'Arrow.

Si l'on se place du point de vue de l'observation, on ne peut manquer de constater qu'il existe des « faits » collectifs qui ne correspondent pas aux propriétés individuelles (pour appartenir à une foule en panique, je n'ai pas besoin d'être moi-même paniqué). On connaît les efforts de G. Le Bon pour aborder la question, même s'il en reste au niveau métaphorique ([1895] 1975, p. 47). On peut dire que la sociologie moderne est née de la réduction de ce type de constatation à sa brutalité de donnée première, en admettant, par principe, le caractère secondaire de l'individu et l'autonomie d'effets qui relèvent de la totalité (Durkheim [1937] 1977, p. 14). La première des règles de la méthode sociologique est de considérer 
les faits sociaux comme des choses. L'individu n'est manifesté que par la contrainte de l'extériorité. Ce modèle s'est imposé, en particulier au travers du courant de pensée structuraliste. Si la sociologie moderne se développe en laissant de côté le problème de l'agrégation, la psychologie de la perception de Köhler et ses disciples, ou psychologie de la forme, évacue directement le Cor. 1-i (on passe des parties au tout par sommation). Plongés dans des environnements différents, les objets ne sont pas perçus de la même façon. Il y a en quelque sorte une «sursommativité », due à la présence de la totalité.

Le rejet du Cor. 1-ii (l'ajout d'une partie change le tout, mais ne change pas les autres parties) a pris plus de temps. Il est principalement dû aux linguistes qui ont étudié la synonymie : aux XVIII et $\mathrm{XIX}^{\mathrm{e}}$ siècles, peu à peu se fait jour l'idée qu'un terme étranger change la signification d'un terme vernaculaire initialement équivalent. Cela aboutira à la valeur saussurienne : un terme se définit par opposition à d'autres (il n'est pas primitif ; contra Cor. 1i). Nous y reviendrons.

\section{LE LANGAGE ET TO}

D'une certaine façon, on peut dire que les sciences du langage sont restées largement insensibles aux problèmes posés par l'infini mathématique à TO et aux discussions autour de l'Ax. 2 (le tout est plus grand que la partie). Trivialement, en matière de langage, le tout, c'est la langue (le français, l'anglais, etc.). Nous reviendrons sur la possibilité que cette totalité soit une réalité empirique. Mais il est clair que l'emploi de notre langage quotidien (le français, est une expression courante et, comme telle, elle n'est ambiguë pour personne) la constitue comme telle, avec l'idée qu'elle a ses limites (ceci ou cela n'est pas français). En laissant de côté le fait qu'il puisse s'agir d'une totalisation imaginaire nous allons nous intéresser aux propriétés qui pourraient concerner les rapports du tout et de la partie.

Si l'on s'attache aux langages formels (un vocabulaire théorique, un vocabulaire terminal, des règles d'engendrement des phrases), il est clair que ces rapports ont un sens au sein de TO : les langages hors contexte sont fermés par rapport aux opérateurs comme « union » ou « intersection ». Outre que nos vernaculaires ne sont manifestement pas de tels langages, quel sens donner à l'union (ou à l'intersection) du français et de l'anglais ? Manifestement aucun qui puisse être opérationnel, puisque nous sommes devant des choix difficiles. $\mathrm{Ou}$ aucun élément ne peut exister qui ne soit strictement du français ou de l'anglais (on ne voit pas l'intérêt de l'union), ou on admet des éléments, disons comme " il est un peu speedé ». Mais on ne peut pas vraiment le faire car cet élément sera conçu comme du français avec création lexicale, importée de l'anglais certes, mais dont la morphologie est bien française et attestée dans des expressions courantes et admises par tous («il est un peu stressé »). On peut tenter de nommer cette 
« partie » de la langue française, le franglais, par exemple. Nous avons d'autres exemples de "parties » d'une langue dans ce que l'on nomme " les langages de spécialité » (Lerat 1995) : le langage des mathématiques, de la physique, etc. Comme le notent les spécialistes (ibid., p. 12), il ne s'agit pas de « sous-langages » au sens technique des langages formels, quoique l'expression " sous-langage » soit couramment utilisée chez Harris et ses disciples (Daladier 1990) dans l'étude des langages de spécialité. On peut donner des caractéristiques générales : morphologie composite, syntaxe typée (comme, dans le cas des mathématiques, pas d'indicateurs de subjectivité, utilisation particulières des modes verbaux, etc.), prépondérance de l'écrit, sémantique particulière, utilisation d'une terminologie propre, présence de signes spécifiques qui ne sont pas des «mots » du français (symboles mathématiques, par exemple). Puisqu'il s'agit de français et qu'une langue spécialisée n'est évidemment pas « tout» le français, il s'agit bien d'une " partie » du français. Mais on n'obtient pas le français en réunissant toutes les langues de spécialité disponibles. Ce ne sont pas des parties au sens propre de la théorie des ensembles. Elles ne sont pas non plus égales au tout, comme les sont les parties infinies d'en ensemble infini : aucune d'entre elles n'est coextensive au français, comme pourrait l'être un de ses dialectes.

On a vu que la notion d'ensemble de tous les ensembles menait à des contradictions. C'est pour les lever que B. Russell a proposé la théorie des types logiques. On ne peut parler d'un langage $L_{n} q u e$ dans un langage $L_{n+1}$. Un langage qui parle d'un autre langage est un «métalangage ». Les propriétés d'un langage donné (ou langage-objet), par exemple celles qui concernent l'expression de la vérité dans ce langage, ne sont exprimables (sans risque de paradoxes) que dans un métalangage. La théorie des types admet pour tout langage une infinité emboitée de métalangages. Le « métalangage » n'est pas simplement une notion abstraite de la logique mathématique. Il apparaît dans les pratiques quotidiennes dès que l'on dispose du discours rapporté, de la nomination de procédés langagiers (« une expression vigoureuse »), de la possibilité de citer ou de donner des noms aux mots et de la référenciation autonyme (« le nom "chien" »). Il est essentiel à la grammaire, puisque cette discipline utilise nécessairement l'autonymie (exemples) et la nomination des catégories et phénomènes linguistiques. Nous connaissons tous des grammaires du français en français. Le langage de la grammaire est évidemment un langage de spécialité. Le français est l'un des métalangages possibles du français, de toute autre langue et de tout autre système symbolique. Contrairement aux langages formels, une « langue quotidienne » (notre français) est un métalangage universel. En ce sens toute langue quotidienne est infinie (comme l'est la hiérarchie des types) et comprend donc une infinité de parties, dont l'une (elle-même) est infinie. Conséquemment, toute représentation d'une langue quotidienne ne peut qu'être parcellaire ; le tout de la langue est irreprésentable. Autrement dit, selon la formule de Lacan, « il n'y a pas de métalangage » (1966), entendons que l'idée qu'il puisse y avoir un système formel ultime représentant 
une langue quotidienne est une chimère ${ }^{1}$.

C'est au logicien Tarski ([1933] 1972) que l'on attribue d'avoir mis en lumière cette propriété de nos vernaculaires. Le linguiste Hjelmslev reconnaît y être parvenu de manière indépendante, dès 1937 :

En pratique, une langue de tous les jours est un langage dans lequel tous les autres langages peuvent être traduits, aussi bien les autres langues que toutes les structures linguistiques concevables. Cette traductibilité résulte de ce que les langues et elles seules sont capables de donner forme à n'importe quel sens ${ }^{2}$. [...] C'est du reste cette propriété qui rend la langue utilisable en tant que telle, et qui la fait propre à remplir son but dans toute situation (1968, p. 148).

Hjelmslev ajoute que « nous n'avons pas à nous demander ici en quoi réside cette propriété remarquable ». Toutefois, il avance une hypothèse intéressante :

Nous sommes enclins à supposer que la raison en est la possibilité illimitée de formation des signes et les règles très libres qui régissent la formation d'unités de grande extension (comme les phrases, par exemple) dans toutes les langues, ce qui, d'autre part, a pour effet de permettre des formulations fausses, illogiques, belles ou morales (ibid.).

On peut accorder au linguiste danois une bonne intuition. Toutefois, l'unicité de « la » raison est une illusion, même si on peut penser qu'il s'agit de cela même que coiffera la première conception chomskyenne de la « créativité ». On se trouve, en fait, face à deux phénomènes qui semblent bien distincts. D'un côté, il s'agit de l'émergence du nouveau. De l'autre, on a affaire à un processus d'extension par « composition » ou d'itération, comme lorsque l'on construit la suite infinie des entiers dans quelque système de numération que ce soit. Nous y reviendrons.

La création du « nouveau »-qui est une propriété du langage déjà remarquée par Descartes dans la dernière partie du Discours de la Méthode (1637) - peut donner lieu à des interprétations « mystiques » à la Humboldt ([1836] 1974, p. 143). C'est un concept difficile à comprendre comme peut l'être l'historicité du monde culturel ou l'évolution des espèces. Pourtant, dans le domaine du langage, nous disposons de la description de mécanismes susceptibles de conforter une approche rationnelle. Nous pouvons en citer un qui provient de l'étude des langues formelles, discipline a priori très éloignée de la question des vernaculaires. Il est disponible

1 On en déduit facilement qu' a fortiori il ne peut y avoir de théorie ultime de toutes les langues (une grammaire universelle), $c f$. Auroux 2009, p. 43 sq.

2 Cela ne signifie pas qu'une langue quotidienne serait une langue universelle, équivalent radical (au sens où l'on parle chez Quine de «traduction radicale ») et redondant de toute langue, exprimant la totalité du réel et dont on peut montrer (Auroux 2009, p. 35 sq.) qu'elle est formellement impossible. D'un côté, la traduction dans une autre langue préserve les idiosyncrasies de chacune (c'est-à-dire l'arbitraire linguistique) : comme le note Quine, la traduction reste indéterminée, autrement que par des hypothèses conjoncturelles (plus ou moins valides) que fait le traducteur. De l'autre, si une langue est apte à exprimer tout ce que nous voulons du monde (elle couvre bien le «tout » du monde), elle le fait de manière non exclusive d'autres possibilités. Si nous le savons, c'est parce que le langage nous est donné dans la diversité des langues. 
dès que S. Lesniewski est revenu en 1928 sur le statut des définitions (voir Zuber 1990). Ce statut, de Pascal à Russell, supposait que, dans un système quelconque (par exemple, en mathématiques, mais aussi dans un langage formel quelconque), on puisse partout substituer la définition au défini salva veritate. Autrement dit une définition ne change rien, elle ne sert qu'à abréger le discours. Ce que Lesniewski découvre, c'est que dans certains cas, l'introduction d'une définition dans un système formel permet de déduire des thèses qu'il était impossible de déduire dans le système initial. La définition agit comme le ferait l'introduction d'un nouvel axiome. Autrement dit le système a changé, il n'est plus le même. L'introduction d'une définition est un acte imprédictible et externe au système, la création d'un nouveau signe. Hjelmslev avait vu juste. Rickey (1975) a démontré que dans un système avec la règle de substitution et de détachement et dans lequel $p \rightarrow q$ est une thèse, aucune définition n'est créative. Mathématiciens et logiciens ne travaillent généralement pas avec des définitions créatives. Dans un système qui dispose de définitions créatives certaines règles dérivées n'appartiennent pas à la règle initiale si l'on y remplace le défini par sa définition. Les langues quotidiennes sont par excellence des systèmes où les définitions sont créatives.

Nous n'avons pas hésité à écrire que nos langues sont infinies. Or il faut être honnête, aucun concept technique mathématique permettant d'appréhender l'infini n'est susceptible d'expliquer ce dont il s'agit ${ }^{3}$. C'est l'auro-référenciation qui introduit cet infini. Certains pourraient préférer le terme « illimité »; mais nous assignons des limites aux langues (« ceci n'est pas du français »). Quel est donc cet objet bizarre que nous nommons sous le terme, bien vague, de langue quotidienne ? Il ne s'agit pas simplement de ce que chacun d'entre nous parle quotidiennement, au besoin avec différents vecteurs, appris en second. Mais de ce qu'il apprend dans son enfance, autrement dit de nos langues maternelles. Une langue quotidienne est donc, avant tout, un système de communication susceptible d'être une langue maternelle. Laissons de côté les problèmes posés par le bilinguisme, voire le multilinguisme ${ }^{4}$. La langue maternelle ce n'est pas simplement le « français langue première » face à « l'allemand langue seconde », c'est ce que j'ai appris dans un parcours évolutif absolument contingent, dans un environnement qui, en partie, n'appartient qu'à moi, puisque nul autre ne peut avoir la même histoire. C'est ce à quoi je ramène mon appréhension de tout autre

3 L'infinité des types logiques que doit contenir la langue quotidienne pour être un métalangage universel fait pencher vers la puissance du dénombrable, mais oblige à accepter la discontinuité comme constitutive du langage, ce que n'hésitent pas à faire la majorité des théories.

4 Nous parlons de l'aspect individuel de la question. Le multilinguisme culturel, lié à des situations historiques particulières (zones de contacts), parfois violentes (invasions des caribes dans les îles peuplées par des tribus arawaks, de famille linguistique différente, avec extermination des hommes et conservation des femmes, entraînant l'existence d'une langue de hommes et d'une langue des femmes - connue des hommes qu'elles ont élevés), ou distribué dans la société en fonction des activités, pose d'autres questions. 
système symbolique, quoique chacun sache que pour parler correctement une autre langue il faut être capable d'éviter de " passer » par sa langue maternelle. D'une certaine façon, on peut penser que le concept lacanien de la « lalangue » est la meilleure approximation que l'on ait fourni du statut restreint que nous venons de donner à la langue maternelle et à son rôle de métalangage universel ${ }^{5}$. On peut interpréter l'hypothèse lacanienne d'un rapport de la lalangue à la jouissance et au désir, comme une façon de formuler l'unicité pour moi (« chacun a la lalangue qui lui est propre ») de ma langue maternelle (il se trouve également que pour la biologie aussi, je n'ai qu'une mère). On comprend ce que peut être la lalangue comme instance dans la réflexion de Lacan et de ses disciples (Assoun 2009), voire la parenté de cette réflexion avec les remarques de Tarski ou Hjelmslev. La spécifier comme objet est autrement plus complexe (Lacan : « le langage est fait de lalangue c'est-à-dire d'une élucubration de savoir sur la langue »). D'autant qu'il est indéniable que si ma langue maternelle est irréductiblement mienne, il n'en demeure pas moins que celle-ci - en l'occurrence le français - est également celle de millions d'autres personnes. Il n'y a pas de langage privé. Si chacun d'entre nous dispose d'une langue maternelle "personnelle », il faut que celle-ci soit différente de celle des autres et que toutes soient du français. Autrement dit, le français n'est pas identique en chacune de ses parties ; la diversité précède l'unité. On retournerait à TO : l'élément (ou la partie) précède le tout. Mais si le français de Jacques n'est pas celui de Paul, comme les deux sont du « français », il faut savoir ce qu'est le français pour pouvoir les identifier. Sans l'idée du tout, il n'y a pas de partie et, par conséquent TO ne saurait être validé dans ce cas.

\section{LA PROPOSITION}

Nous avons déjà noté qu'avec l'alphabet, les sciences du langage occidentales ont donné un modèle (artefactuel) pour la discussion tout/partie. Un ensemble fini d'éléments permet d'engendrer ad libitum les composés que sont les mots ou les énoncés. Ce modèle implique donc la notion de compositionnalité, qui n'est rien d'autre qu'une façon opérationnelle de formuler le Cor. 1-i. Toutefois le tournant essentiel des sciences du langage occidentales concerne l'appréhension de l'énoncé ou de la proposition (Auroux 2008), ce que les grecs nommaient le logos.

Ce tournant date du dialogue Le Sophiste et de la formulation de ce que je nomme (Auroux et al. 1997 ; Auroux 2008, 2009) « le théorème de Platon » en

5 « Car les logiciens - les plus subtils ne s'en cachent pas -, c'est de la lalangue elle-même pour peu que l'existence s'en atteste, qu'ils font la limite totalisante des langages logiques ; il suffit pour cela qu'un nom soit trouvé - langue quotidienne de Tarsky, langue U de Curry - qui la fixe à sa position » (Milner 1978, p. 79). 
$261 \mathrm{~d}$ et dans les pages suivantes. Dans un langage proche de celui du philosophe, on peut formuler ce théorème de la façon suivante :

Une suite de signes n'est pas une proposition (logos) ; pour qu'il y ait langage, il faut qu'il y ait la présence d'au moins deux types de signes, onoma (nom, thème) et rhêma (verbe, rhème), ou plus généralement il faut que des signes puissent occuper deux types de fonction.

La démonstration, comme souvent chez Platon, se fait par ostension et généralisation, sans oublier la preuve par contre exemple. Il est évident que l'analyse repose largement sur le marquage morphologique de l'opposition verbonominale des langues indo-européennes. On ne peut reprocher à Platon de ne pas connaître le chinois et les langues isolantes, ne disposant pas ou quasiment pas de morphologie. Il n'en demeure pas moins que sa position est totalement généralisable. Elle tient à la mise au jour d'une propriété essentielle du langage humain qu'on pourrait appeler son caractère " propositionnel ». Même si l'on peut éprouver quelque difficulté à définir ce qu'est une " proposition", on voit bien de quoi il s'agit. Pour qu'il y ait du langage humain, il faut pouvoir dire quelque chose de quelque chose. En même temps, ce qui est clairement énoncé, c'est que n'importe quel élément de langage ne peut pas être simplement ajouté à n'importe quel élément pour qu'il y ait proposition. Nous dirions aujourd'hui que la composition du tout de la proposition ne peut pas être une simple concaténation, il faut des règles, une syntaxe. Dans l'optique des langues européennes, le théorème de Platon revient à dire que pour qu'il y ait proposition, il faut que les éléments soient « arrangés » en fonction de leur appartenance à des catégories différentes. On sait aujourd'hui que ce n'est pas tout à fait une exigence universelle, puisqu'il existe ce que Launey (1994) nomme les " langues omni-prédicatives », où, comme pour le nahuatl, les éléments de toutes les catégories peuvent apparaitre en position de prédicat. Mais il n'existe pas de langue sans règle et sans proposition. Le passage des parties au tout, tel qu'il s'effectue dans cet aspect des langues ne dispose d'aucun modèle dans le monde naturel. Il y a des formes de relations entre les parties, par exemple, ce que les générativistes nomment la c-commande6 ou ce que l'on nommait autrefois l'accord et le régime, qui sont propres au langage humain. Si l'on suit le développement historique de la description des différentes parties du discours (Colombat [dir.] 1988 ; pour une approche contemporaine, voir Le Maréchal 1989) on ne peut que remarquer la nécessité de partir d'une unité préalable, le fameux discours, qui est un présupposé indispensable. C'est ce que Denys le Thrace remarque dans la Tekhnê grammatikê, la première grammaire du grec (ancien) aujourd'hui disponible (II siècle avant notre ère) :

6 On dit qu'un élément A c-commande un élément B si A ne contient pas B et est dominé par la première catégorie branchante qui domine $\mathrm{B}$. Autrement dit : $\mathrm{C} \rightarrow \mathrm{A}, \mathrm{B}$, c'est-à-dire Phrase $\rightarrow$ Pronom, verbe $\rightarrow$ Il mange, mais aussi GN (groupe nominal) $\rightarrow$ N (nom), ADJ (adjectif) $\rightarrow$ cheval blanc. 
Le mot (lexis) est la plus petite partie de la phrase (logos) construite. La phrase (logos) est une composition en prose qui manifeste une pensée complète. La phrase a huit parties : le nom, le verbe, le participe, l'article, le pronom, la préposition, l'adverbe et la conjonction (traduction J. Lallot 1989, p. 49).

Au premier abord le mot provient de la fragmentation de l'énoncé. Le concept (l'un des termes théoriques essentiels de la grammaire occidentale) a connu une histoire compliquée quant aux tentatives de le définir. On peut l'aborder par la liste des contraintes qui pèsent sur la fragmentation en question :

i) les mots doivent être des signes, c'est-à-dire posséder une signification, même si celle-ci n'est déterminée qu'en contexte (les lettres ou les sons ne sauraient être des mots en eux-mêmes);

ii) la chaîne linguistique (l'énoncé, la proposition, le logos) est un arrangement de mots qui doivent par là posséder une certaine autonomie (ils figurent comme tels dans les dictionnaires) ; les mots sont les éléments (les atomes) de l'expression linguistique ;

iii) s'il faut faire quelque chose en plus de l'arrangement des mots pour obtenir une expression linguistique, cette modification est toujours conçue comme une modification interne du mot.

Les deux premières contraintes correspondent à ce que l'on peut déduire de TO et de la compositionnalité. La troisième est moins évidente. Elle revient à dire que rosarum n'est pas un « mot», c'est la forme fléchie au génitif pluriel du « mot » dont le nominatif singulier est rosa. Une flexion est toujours la flexion d'un mot ; les deux concepts sont interdépendants : la forme fléchie n'est que l'élément d'un paradigme servant à caractériser une espèce de mot. Une partie du discours (une classe de mots, comme celle des verbes), quant à elle, correspond à un type de flexion spécifique (la conjugaison pour le verbe, la déclinaison pour les noms). Que la grammaire occidentale répartisse exhaustivement les mots dans différentes classes et s'en serve pour construire la représentation des constructions grammaticales conduit à la distinction entre flexion et dérivation. Singh \& Ford (1980) notent qu'un morphème est une flexion (et non un affixe dérivationnel), s'il est membre d'un paradigme de formes servant à caractériser une partie du discours. C'est pourquoi dans la grammaire antique, le participe est classé comme une partie du discours distincte du nom et du verbe. À l'inverse des morphèmes latins comme -ans, $-u m$ ou -ndus ne sont pas en latin considérés comme des flexions, mais comme des dérivations, parce que, respectivement, le participe présent, le supin ou le gérondif ne sont pas conçus comme des parties du discours indépendantes. La tradition sanskrite se passe de la distinction entre flexion et dérivation : « pour Panini, il n'y a que des affixes, qui se distinguent seulement par leur rang dans la chaîne de dérivation » (Pinault 1989, p. P374).

On remarque que les mots font l'objet d'une double approche, soit on les considère comme les éléments d'une proposition $(\log o s)$, soit on les considère 
comme les éléments qui appartiennent aux différentes étapes d'une classification au sens strict. Cette seconde approche permet d'aborder la question de leur universalité ; les latins savaient bien que, contrairement au grec, leur langue ne possédait pas d'article. Au XVIII' siècle, le grammairien Beauzée admet la diversité en posant que dans la classification des mots il existe un niveau de branchement sous lequel doivent apparaître toutes les classes connues ou possibles de toutes les langues. La classe « article » n'est pas universelle, elle se branche sous la classe « adjectif » dont elle est l'un des nœuds ${ }^{7}$.

Il résulte de tout ce qui précède que quand bien même le mot est conçu comme un élément consistant et quasi naturel du tout qu'est l'énoncé, il n'en demeure pas moins qu'il s'agit d'une construction théorique due à nos grammairiens. Cette construction semble extrêmement productive dans la conception des langues indoeuropéennes et elle a pour corolaire la forme de nos dictionnaires, qui sont des listes de « mots » au sens que l'on vient de décrire ${ }^{8}$, une fois que l'on a choisi la forme canonique du représentant du paradigme. On sait les difficultés rencontrées par les linguistes lorsqu'il s'est agi de décrire des langues agglutinantes ou « polysynthétiques » (phénomène de l'incorporation ${ }^{9}$ ). Dans l'écriture de son premier dictionnaire caribe-français (1665) l'abbé Breton avoue ne pas savoir très bien à quelle « lettre » classer certains « mots »; entendons qu'il a quelque mal à définir ce qui est un mot et ce qui ne l'est pas (affixe). Les structuralistes américains ont choisi de se passer des «parties du discours » au profit d'une catégorisation interne à chaque langue, repérée par les méthodes distributionnelles. Dans son célèbre article de 1954, Hockett distingue trois modèles d'analyse grammaticale. Il laisse de côté celle que nous venons de décrire (qu'il intitule " word and paradigm ») au profit de deux modèles structuraux, l'un morphématique (« item and arrangement »), l'autre dynamique ("item and process »). On peut dire que face à la question séculaire des catégories, les structuralistes ont choisi de faire de la linguistique d'abord une procédure inductive. Il s'agit de " découper » la chaîne énonciative, de reconnaître l'identique et de regrouper par classes. Nous savons qu'en dehors d'un univers fini, et même relativement petit, il n'existe pas de « machine inductive ». À un moment ou à un autre, les linguistes doivent donc

7 Pour sauver l'universalité on n'est pas obligé d'adopter une politique de classification. On peut considérer les classes comme des éléments susceptibles de composition. La contrainte d'universalité est satisfaite si l'on trouve le plus petit nombre d'éléments dont la composition permet de reconstruire toutes les classes. Ainsi, la classe « adverbe » n'est pas un universel si on admet « adverbe = préposition + nom ». Condillac, à l'encontre de son contemporain Beauzée, n'admet que trois éléments " universels », le nom, la préposition et le verbe substantif être.

8 On peut imaginer les remplacer par des listes de racines ou de morphèmes.

9 Il faut attendre Baker 1988, pour voir le phénomène traité par la grammaire générative. Même dans ce cadre, cela suppose de notables amendements. Comme le note Chomsky 1995, p. 182-184, le nœud dominant de la dérivation n'est plus la Phrase (P), mais un terme nouveau AGRP, ce qui revient au vieux concept d'holophrase. 
faire le « saut » de l'induction, ce que le philosophe Goodman (1983) nomme une " projection ». Nous voulons dire par là que la classe, aussi distributionnaliste que soit son approche dans un corpus aussi grand que l'on veut, ne peut être seulement définie comme la somme d'éléments observés en certaines positions, elle doit valoir pour (pouvoir être projetée sur) des éléments non observés. De ce point de vue, les parties du discours traditionnelles dans leur présupposé universaliste ont une « bonne valeur projective », même si cela ne marche pas toujours très bien ! Quoi qu'il en soit, il semble que toutes les approches du langage humain reposent sur cette idée qu'il y a des parties élémentaires (" plus petite partie de l'énoncé » disait Denys du mot), des éléments discrets, dont les arrangements forment à volonté des énoncés. Nous respectons TO et le modèle de l'alphabet paraît le bon.

La totalité de ces parties, c'est donc l'énoncé. Comme on l'a vu, selon Denys, le critère de l'énoncé est de manifester " une pensée complète ». Empiriquement, il n'est pas très clair de savoir ce qu'est une " pensée complète ». On peut dire, cependant, qu'il s'agit nécessairement d'un tout fini qui possède des parties; le manque d'une de ces parties correspond à l'incomplétude du tout, donc au fait que l'on n'est pas en présence d'un « énoncé ». Platon procédait par ostension, ceci (« exemple ») est ou n'est pas un énoncé. Pour parlez il faut bien que nous sachions ce qui est ou n'est pas un énoncé. L'une des propriétés essentielles du langage humain est de permettre la communication, c'est-à-dire le dialogue. Le " tout fini », est quelque chose d'achevé à quoi je puis répondre par un autre tout fini, qui, une fois achevé, permet éventuellement une réplique. Bien entendu, nous pouvons laisser des " trous » dans nos touts finis énonciatifs, mais c'est à condition de pouvoir les remplir, donc à l'aide d'une image potentielle du tout fini. De fait, logiciens (Aristote, puis les stoïciens) et grammairiens ont construit une représentation de l'énoncé à l'aide des catégories qu'ils construisaient. On sait que le modèle aristotélicien était «Sujet copule Prédicat». Ce n'était ni le seul possible, ni le seul qui fut proposé au cours de l'histoire. Tout comme en logique il rendait impossible la représentation des relations, en grammaire il bloquait la conception du verbe et de la complémentation. L'utilisation de la notion de sujet par les grammairiens ne remonte pas avant le $\mathrm{XI}^{\mathrm{e}}$ siècle de notre ère. $\mathrm{Ce}$ n'est qu'au XVII' siècle (par exemple dans la Grammaire de Port-Royal) que ces derniers commencèrent à utiliser la notion de proposition. Dès lors était engagé le processus par lequel on allait être conduit à reconnaître deux types de composition dans un énoncé ; celle qui concerne ses " parties logiques » (le fait qu'il y ait sujet et prédicat) et celle qui concerne ses " parties grammaticales » (la composition des parties logiques en terme de «mots »). Une proposition peut contenir d'autres propositions. Par exemple, elle peut contenir deux sujets ( $\mathrm{S}_{1}$ et $\mathrm{S}_{2}$ sont $\left.\mathrm{P} »\right)$ et, par conséquent, donner lieu à deux propositions, chacune ne conservant que l'un des sujets. La composition grammaticale concerne la façon dont les éléments logiques sont réalisés linguistiquement. On peut déterminer une composition minimale. Par exemple, un « Nom commun » ne peut pas rester seul en français, il faut lui 
adjoindre un article ou un quantificateur. De plus, on peut lui ajouter un qualificatif comme « expansion ». Une proposition peut contenir d'autres propositions dans sa composition grammaticale, qu'elles soient relatives ou subordonnées. Les expansions accroissent la taille concevable de l'énoncé conçu à partir d'une structure élémentaire de base.

Le passage à la grammaire formelle, donc à l'idée d'engendrer des énoncés à partir d'un vocabulaire initial fini et de règles en nombre fini, nous fait passer de l'expansion à l'itération (la récursivité). À première vue, personne n'a jamais formulé de principe pour fixer explicitement une limite à l'expansion, c'est-à-dire pour préciser le maximum de parties que pouvait avoir la totalité énonciative. Les tenants de la grammaire formelle (Chomsky 1990) ont posé que l'itération ne pouvait avoir une telle limite et qu'elle était la base d'un caractère essentiel du langage humain, son caractère infini :

(...) toute langue naturelle comporte un nombre fini de phonèmes (ou de lettres dans son alphabet) et (...) chaque phrase peut être représentée comme une suite finie de ces phonèmes (ou lettres) bien que le nombre des phrases soit infini (Chomsky [1957] 1969, p. 15).

Entendons que l'ensemble des phrases d'une langue a la puissance du dénombrable. Il dispose donc de parties infinies de même puissance. On a beaucoup critiqué (Parkinson 1972 ; Auroux 1998, p. 92-97) cet aspect de la théorie, auquel Chomsky n'a jamais renoncé (1995, p. 14-15). Je pense que l'idée triviale du linguiste selon laquelle l'itération expliquerait la créativité linguistique ne mérite plus guère d'être mentionnée, même si une telle conception a été la base de l'ars inveniendi leibnizien. Programmez un ordinateur dans une cave pour engendrer la suite des entiers naturels, mille milliards d'années plus tard il n'aura toujours pas engendré un nombre irrationnel, ce qui, dans ce contexte, serait la seule véritable marque de créativité. Remarquons que Chomsky ne soutient évidemment pas l'existence de phrases de longueur infinie : comme les entiers naturels les phrases sont toutes finies, ce qui signifie que quelle que soit la taille d'une phrase donnée, on en trouvera toujours une plus grande. Mais par principe, il faut donc admettre dans la grammaire des phrases de longueur « illimitée ». Cela va à l'encontre autant de l'intuition (mais les générativistes ont tendance à croire que la contre-intuitivité d'une assertion est la marque de sa scientificité !) que de toute la tradition grammaticale.

On connait la réponse, maintes fois réitérée, de Chomsky. Elle tient dans la distinction entre la compétence et la performance. Nous reviendrons sur ces notions dans la section suivante. L'idée que c'est la " performance » qui limite la longueur des phrases (à cause des possibilités mémorielles) permet de préserver l'idée que cette limitation n'est pas une propriété appartenant en propre au langage. Paradoxalement, on peut dire que Chomsky a refusé la solution que les grammairiens ont privilégiée depuis l'âge classique. 
Pour les classiques, héritiers en cela du subjectivisme cartésien, toute pensée, dont le langage est l'expression, est un acte fini et instantané. Il y a toujours un « je pense » derrière un contenu de pensée et, par conséquent, un sujet derrière toute énonciation. Cela signifie que la valeur d'un énoncé et son unité tiennent à l'intention de celui qui l'énonce. Les Messieurs de Port-Royal utilisent cette notion pour maintenir la distinction entre relative déterminative et explicative (appositive) quand il n'y a rien dans les formes qui puisse permettre de les distinguer. L'idée d'un sujet de l'énonciation est aujourd'hui indispensable à la compréhension, notamment, des pronoms personnels Je/Tu (Il étant considéré comme une non-personne). Cette introduction du sujet de l'énonciation appelle plusieurs remarques. D'abord, nous pouvons distinguer clairement la façon dont il s'inscrit dans nos langues ( $c f$. Benveniste : l'appareil formel de l'énonciation), le philosophe américain W. V. O. Quine allant même jusqu'à suggérer (à juste titre) que sans cet aspect de nos langues il n'y aurait pas de détermination possible de la référence (1960). Ensuite, cela conduit à reconnaître une autre dimension du fonctionnement linguistique. Jusqu'ici nous étions restés dans l'ordre symbolique des formes. L'énonciation nous oblige à ajouter la dimension imaginaire. Pour comprendre ce que l'on me dit je dois avoir une idée de l'intention de mon interlocuteur. Les éthologues font remarquer depuis plusieurs années qu'il manque avant tout aux autres animaux pour posséder le langage, c'est la capacité de se représenter l'esprit d'autrui.

L'énonciation est l'acte d'un sujet situé dans l'espace/temps. Arrêtons-nous sur ce point. Soit un énoncé : « Le rossignol est revenu ». Tel qu'il est écrit là sur la page, il est simplement un signe. Un signe soit, mais de quoi ? C'est un exemple comme l'on en trouve dans toutes nos grammaires. Autrement dit, il est le signe de toute énonciation qui lui sera homophone ; il est également autonyme de luimême. Katz (1981) soutenait pour cette raison que ce genre de chose était un universel semblable aux idées platoniciennes. C'est absurde parce que pour cela il faudrait qu'il puisse déterminer ce que signifiait l'énonciation de ce " même » énoncé, tel jour de l'année dernière, dans mon jardin, en présence de mon petitfils. Or, ce qui déterminait la signification c'était lui et moi, et le soleil et le jardin. Autrement dit le monde réel. Cela fait partie de ce que je nomme « l'externalité de la référence » (Auroux 1998, p. 117 sq.). Évidemment, je peux paraphraser ou retenir quelque chose de cette référence dans mon langage quotidien ; par exemple, " c'est le printemps, il fait chaud » ou « le printemps est précoce cette année » ou « il est temps de construire le nichoir promis ». Mon langage peut couvrir toutes les situations, tout le réel. Mais contrairement à ce que pensait le premier Wittgenstein, ce n'est pas mon langage qui est la limite de mon monde, c'est mon monde qui est la limite de mon langage, parce que c'est lui qui lui donne un contenu. Changer de monde, comme l'ont fait les colons de la Renaissance, c'est changer de langue sans cesser de parler sa langue maternelle. Un « grand arbre » ne pouvait avoir la même signification pour un portugais et un brésilien quand bien même ils 
parlaient la même langue. On a discuté sans fin de l'universalité ou du relativisme (attesté) des catégories de couleur dans les langues. C'est oublier que les noms de couleur n'apparaissent pas abstraitement, mais sont d'abord référenciés à partir des objets du monde environnant, parfois artificiels, comme les nuanciers pour les papiers peints, les vernis à ongle, les parfums (Dubois 2009) ou les fils d'un métier à tisser (Cardona 1985, chap. 9, p. 164 sq.). Si l'on reste dans la nature, le « vert » n'est pas à proprement parler une signification universelle, puisque sa signification ne saurait être la même pour un pygmée de la forêt et pour un habitant du désert. Contrairement à ce que soutiennent Berlin \& Kay (1969) l'apparition des termes de couleur dans une culture donnée ne suit pas un ordre universel objectif, mais obéit aux aléas du développement culturel, ils « adhèrent » à des éléments distingués de l'environnement (Cardona $1985 ; 1990)$. Pour comprendre la totalité du phénomène linguistique, il faut ajouter le réel (qu'il s'agisse de la nature comme de la culture) à l'imaginaire et au symbolique. L'externalité de la référence et sa participation à la détermination des éléments linguistiques est telle qu'un individu abandonné dans une île déserte et n'ayant de contact avec une langue inconnue que par une radio monolingue serait incapable de la comprendre et, bien entendu, de l'apprendre. Comme le notait Wittgenstein, une langue est une forme de vie. Pour parler, il faut avoir un accès au monde et à autrui.

L'énonciation, l'acte langagier essentiel, met au jour une propriété nouvelle du langage que l'on peut qualifier d'absence de compositionnalité. Comme on l'a vu, la théorie de l'énoncé mise au point par les grammairiens, repose sur la composition $\mathrm{du}$ tout par des parties. Le sens d'une énonciation dépend du contexte, pris comme un tout global avec l'énonciation elle-même. Il n'y a de sens linguistique que dans l'acte d'énonciation qui lie irrémédiablement symbolique, réel et imaginaire. Le langage est cependant une réalité symbolique un peu particulière. Il peut en effet être "représenté » de maintes façons en utilisant des parties de lui-même. On connaît les multiples supports transposés possibles pour de nombreuses langues : langages tambourinés, sifflés ou systèmes d'écriture, etc. À chaque fois ce n'est pas la « totalité » du langage telle qu'elle se révèle dans l'acte d'énonciation qui se trouve présente dans le support transposé. On connait les réactions de Platon, qui dans le Phèdre, voyait dans l'écriture une véritable « mutilation » par rapport à l'oral. Plus personne ne pouvait répondre de son logos en face à face. La grammaire - qui, il est vrai dépend de l'écriture (Auroux 1994) - est aussi une représentation « partielle ». Elle l'est d'abord parce que l'exemple que l'on y rencontre ne peut qu'être une partie de l'énonciation. Elle l'est ensuite, parce que comme l'ont montré les historiens modernes des sciences du langage (Auroux 1998, p. 185 sq. ; Fournier [dir.] 2007), les corpus d'exemples sur lesquels elles reposent ne sont pas des «données brutes ». Ce sont des constructions qui peuvent venir d'un choix puriste ou du dispositif théorique lui-même, le plus souvent des deux ; ces constructions s'héritent au sein d'une même tradition grammaticale, voire d'une tradition à l'autre. Mais personne ne soutiendra sérieusement que les exemples 
d'une grammaire, quand bien même le but de cette grammaire est d'apprendre à parler « une » langue, soient la « totalité » de la langue. Dans une grammaire, nous travaillons avec une " partie distinguée » que nous jugeons représentative de la totalité. On peut imaginer que les « règles » (ou " théorèmes » de la grammaire, quel que soit le nom qu'on leur donne) n'ont pas ce défaut de partialité, puisqu'elles sont censées être générales. Au reste, il suffit de disposer d'une liste de mots pour pouvoir les substituer aux éléments de l'exemple et « agrandir» le corpus. Mais encore un coup, quel que soit l' " agrandissement», on n'atteindra jamais la totalité des énoncés qui surviennent dans l'échange langagier. C'est le phénomène auquel j'ai donné le nom de sous-détermination grammaticale (Auroux 1994). Pour donner une image maximale du phénomène, disons qu'on pourra manipuler tant qu'on veut les exemples et les règles d'une grammaire latine, en respectant les prescriptions de cette dernière, on n'obtiendra jamais un énoncé d'ancien français. La sous-détermination grammaticale est cohérente avec la conjecture de TarskiHjelmslev. Une grammaire « totale » d'une langue quotidienne serait celle d'un métalangage universel, elle devrait donc elle-même être une langue universelle sans être une langue quotidienne, ce qui est contradictoire.

On peut se poser la question de savoir ce qu'est véritablement un « exemple». Quand j'énonce quelque chose, mon énonciation peut être " conforme » à un exemple grammatical (du moins c'est ce que l'on explique à l'école !). Littéralement, les éléments sont identifiés comme les «mêmes ». Pour comprendre l'identité élémentaire ou l'universalité qui fait que ceci vaut pour cela, il n'est nullement nécessaire de postuler quelques entités mystérieuses susceptibles de se manifester identiques à elles-mêmes dans toutes les circonstances. L'identité ou universalité élémentaire c'est, comme le remarquait Locke (Auroux et al. 1996, p. 88), la non distinction. Un atome d'hydrogène est la « même chose » qu'un autre atome d'hydrogène parce qu'il lui est indéfiniment substituable. Pareillement, n'importe quelle pièce d'une certaine valeur que j'ai dans ma poche fera l'affaire dans le distributeur de boissons. Dans une grammaire, un exemple est le signe de lui-même (dans cette occurrence là et dans toutes les autres pareillement), il « suppose » matériellement comme disait encore Leibniz en reprenant la terminologie médiévale. Les parties d'une langue servent éventuellement de signes pour elles-mêmes ou ce qu'elles représentent.

L'énonciation (ou plus simplement l'acte de langage, ici et maintenant dans l'activité langagière) est évidemment unique, comme l'est un élément de matière dans l'espace-temps. Lui seul possède ce qu'il convient d'appeler une signification au sens plein. Tous ses éléments n'obéissent pas à la loi de compositionnalité, il est clair qu'une perception globale du contexte entre souvent en ligne de compte. C'est une propriété du langage, cohérente avec celles que l'on vient de décrire que l'on puisse en « extraire » des éléments, comme ces fragments de langage que l'on utilise comme exemples. Les grammairiens ont pris l'habitude de cette décomposition et de cette abstraction au cours de laquelle on doit laisser beaucoup de choses de côté. 
Le caractère discret des éléments sonores (le modèle artefactuel le plus utilisé de l'élément d'un tout, comme on l'a vu) procède de cette abstraction, dont la réalité concrète n'est pas toujours évidente (Albano Leoni 2009). L'idée de composants du langage (par exemple, composant phonétique, syntaxique et sémantique) n'est pas nécessairement aberrante si l'on admet qu'il s'agit d'une abstraction commode des grammairiens. Leur réalisme (Fodor 1987) est plus discutable.

La thèse de l'autonomie de la syntaxe est source de réels problèmes philosophiques et empiriques. Une structure syntaxique c'est pour le théoricien une formule littérale comme l'était le « $\mathrm{S}$ est $\mathrm{P}$ » de la logique traditionnelle, ou le $« \mathrm{P} \rightarrow \mathrm{SN}, \mathrm{SV} » \mathrm{~d}$ 'une grammaire de constituants sous forme de règles de réécriture. Mais y a-t-il lorsque nous parlons quelque part dans notre esprit un accès direct à de telles formules littérales? Pour manipuler un vernaculaire faut-il que notre esprit soit subrepticement équipé d'un langage interne formel (i-langage), un peu comme les ordinateurs disposent d'un langage résident? Fonctionnons-nous sur un mode digital ou plus précisément sur un mode analogique ? Par " mode analogique » de fonctionnement, j'entends, en matière de langage, une manipulation de formes linguistiques comme sont les exemples. Je suis persuadé qu'aucun formalisme n'est à la source de nos langues quotidiennes, c'est au contraire le développement de ces dernières, dans des contextes culturels précis et selon une histoire complexe, qui a rendu possible la naissance des systèmes formels les plus sophistiqués.

Si l'on suit l'histoire des mathématiques, le calcul abstrait le plus élémentaire, n'est ni une donnée primitive ni une donnée interne à l'esprit. Il a d'abord fallu que soit donnée valeur symbolique à des éléments externes : parties du corps, petits cailloux (lat. calculus). Puis on a créé des instruments techniques totalement ad hoc comme les bouliers. Enfin, l'écriture a permis l'existence de formules représentant et effectuant l'activité du calcul. Ajoutons que le formalisme, au sens strict du terme (ici, nos formules littérales) ne requiert en rien la littéralité, il peut être manifesté dans nos vernaculaires. Pendant des siècles, la logique formelle a fonctionné sans « formalisme » (de façon analogique), en manipulant de manière réglée des expressions canoniques homophones à des formules (évidemment choisies) de la langue quotidienne, même si ces formules peuvent paraître un peu gauches aux utilisateurs de cette dernière (Auroux 1993, p. 30-31). Il y a quelque chose de paradoxal à imaginer le langage d'abord enfermé dans une compétence innée purement abstraite et intellectuelle. Comme si, pour l'être humain, le langage n'était pas d'abord une façon d'instrumentaliser son propre corps ${ }^{10}$ et son environnement. La faculté du langage n'est qu'un « après-coup » de l'invention du

10 Le sociolinguiste Cohen remarquait déjà l'importance des techniques corporelles dans l'expression langagière : "Technique du corps et invention intellectuelle, le langage parlé est incorporé au fonctionnement physiologique de la vie normale de l'homme en société » (1972, t. 2, p. 110). Sans négliger cet aspect, nous allons plus loin, en suggérant que parler c'est transformer son propre corps en porteur de significations. 
langage. Comme il faut des mathématiques pour qu'existe la faculté de résoudre des équations, il fallait bien qu'il y ait du langage pour qu'existe une faculté du langage.

\section{LA LANGUE}

Si la proposition est une totalité dont l'unité s'exprime empiriquement dans un acte concret, la langue est quelque chose de difficile à saisir empiriquement. Dans la plupart des groupes humains où la communication fonctionne, on se forge une représentation plus ou moins imaginaire de ce vecteur de communication, dont l'image participe fortement à l'identité du groupe. Mais la communication ne saurait fournir la base d'une construction stricte de la langue : comme la « compréhension » n'est pas transitive ( $\mathrm{Si}$ A comprend B qui comprend $\mathrm{C}$... qui comprend $\mathrm{N}$, il ne s'ensuit pas que A comprenne $\mathrm{N}$ ), elle ne saurait fournir la relation nécessaire à la constitution d'une classe d'équivalence. La première solution qui vient à l'esprit est la sommation, ne serait-ce que celle des mots. Beauzée dans l'article " langue » de l'Encyclopédie est un peu plus précis. « La langue est la totalité des usages propres à une nation pour exprimer ses pensées par la voix ». Mais on ne va guère plus loin qu'une définition nominale, d'autant que le concept de « nation » est loin d'être clair.

La seconde solution consiste à chercher des « faits de totalité», autrement dit des « faits de langue ». On peut évidemment dire quelque chose comme « contrairement au grec, le latin ne possède pas d'article ». Cette généralisation, en la complétant d'autres du même genre pourrait permettre de caractériser effectivement telle ou telle langue prise dans sa globalité. Peut-être même suffisamment pour la reconnaître. On ne serait plus dans la sommation des éléments, mais dans celle des propriétés. N'y aurait-il pas quelque phénomène qui affecte la « langue » dans son unité de totalité?

Le comparatisme a approché la question. Depuis la Renaissance, l'histoire des langues était abordée de façon " atomique ", à partir du mot, unité de référence, et de ses éléments, les « lettres » (les phonèmes). Dans un mot donné les lettres peuvent permuter (métaplasme, par opposition au trope figure de signification) pour des raisons arbitraires et donner un autre mot (au besoin d'une autre langue). Cette technique de permutation, dont l'origine remonte à l'Antiquité, a permis à Hickes (Institutiones grammaticae anglo-saxonicae et moesogothicae, 1689) de rédiger la première grammaire d'une langue morte (celle de la Bible d'Ulphila), en la comparant au vieux saxon. Ce sont les mots qui sont comparés et identifiés, aux permutations près. Ce qu'apporte Grimm en 1822, dans la seconde édition de sa Deutsche Grammatik, c'est un changement de référentiel. Pour décrire la Lautverschiebung, la mutation consonantique qui sépare les « langues germaniques » des autres langues indo-européennes, la référentiel n'est plus le 
mot dans sa singularité, mais toutes les occurrences du phonème dans l'ensemble de la langue.

La référence à la langue dans sa globalité est par définition l'une des caractéristiques de la grammaire comparée et historique. La discipline a pour tendance naturelle de privilégier un modèle arborescent et discontinuiste de l'ensemble des langues, c'est-à-dire le vieux modèle généalogique, déjà présent dans l'Ancien Testament. L'innovation, au xviiie siècle était plutôt la prise en compte d'un modèle diffusionniste (Bertrand 1758). Avec la prédominance du comparatisme, il faudra attendre les années 1880 et la dialectologie moderne pour remettre en cause le discontinuisme (parfois appuyé sur l'idée que les langues naissent et se développent comme des plantes). Du coup l'identité de la langue comme totalité refait problème. Pour les romanistes français (P. Meyer) de la fin du XIX siècle, elle n'est pas une " espèce naturelle », il n'y a pas de véritable frontière dialectale, tout au plus peut-on tracer des isoglosses. Entre le continuisme et le discontinuisme, nous sommes, comme le notera V. Henry en 1897, en face d'une véritable antinomie linguistique. Cette dernière remet en cause la réalité de la langue comme totalité.

Tout au long du $\mathrm{XIX}^{\mathrm{e}}$ siècle les linguistes ont stabilisé une invention des grammairiens du XVIII. Il s'agit d'une nouvelle interprétation du phénomène de la synonymie (Gauger 1973 ; Auroux 1984, 1986). Deux mots synonymes ne signifient pas exactement la même chose, ils doivent aussi manifester des différences : "L'inclination dit quelque chose de moins fort que le penchant. La première nous porte vers un objet, et l'autre nous y entraîne » (abbé Girard). Dès son Traité des Tropes de 1730, C. Dumarsais notait l'impossibilité qu'existent des mots parfaitement synonymes dans la même langue. L'impressionnante série des dictionnaires français de synonymes (qui se reprennent et se complètent et que l'on adapte parfois en d'autres langues comme l'espagnol et le russe) développe ce que l'on appellerait aujourd'hui une véritable analyse sémique. B. Lafaye en a fait l'histoire et la théorie, dans l'édition remaniée de 1861, de son dictionnaire des synonymes qui avait obtenu le prix linguistique (Prix Volney) de l'Institut de France en 1843. Un point important est que les auteurs ont très vite remarqué que l'introduction dans une langue d'un synonyme issu d'une langue étrangère, conduisait immanquablement à produire une différence entre le mot de la langue et celui d'origine étrangère. Ils en donnent des exemples dans plusieurs langues que ne manque pas de reprendre largement un compilateur acharné comme R. de la Grasserie $^{11}$. Par rapport à la problématique de la totalité, on assiste à une véritable

11 Grasserie 1908 : « Le mot anglo-saxon indique le bœuf sur pied ; le mot anglais beef le bœuf abattu ; la même distinction existe entre : calf et veal, lamb et mutton » (p. 107 et 264). J'ai choisi ces exemples parmi une multitude parce que ce sont ceux que l'on trouve chez Saussure. «Le français mouton peut avoir la même signification que l'anglais sheep, mais non la même valeur, et cela pour plusieurs raisons, en particulier parce qu'en parlant d'une pièce de viande apprêtée et servie à table l'anglais dit mutton et non pas sheep. 
invention, puisque le Cor. 1-ii qui assure que l'ajout d'un élément ne change rien aux autres est invalidé.

C'est un point qui n'a pas échappé à Saussure :

Dans l'intérieur d'une même langue, tous les mots qui expriment des idées voisines se limitent réciproquement ; des synonymes comme redouter, craindre, avoir peur ${ }^{12}$ n'ont de valeur propre que par leur opposition ; si redouter n'existait pas tout le contenu irait à ses concurrents (Cours..., p. 160).

S'il est certain que le genevois s'inspire d'un traitement classique d'un phénomène linguistique (Auroux 1985), il fait preuve d'un « culot» théorique extraordinaire en affirmant que l'opposition (la différence) est la réalité même de l'élément linguistique, sa valeur. Dans une langue il n'y a que des différences, elle est forme et non pas substance.

Il y a donc quelque chose de systématique qui nous permet de considérer l'effet de certains éléments d'une langue sur d'autres. S'ensuit-il qu'une langue soit un système où tout se tient, comme l'affirme Saussure dans une phrase du Cours, restée célèbre ? Dans ce cas le tout devrait être donné pour que soient données les parties ${ }^{13}$. Comment le tout peut-il être donné ? Doit-on admettre avec Lévi-Strauss (1950, p. XLVII), que l'univers serait devenu signifiant d'un seul coup ? Saussure a été assez obscur sur ce point. Il développe l'idée que le signe linguistique mettrait en relation deux masses amorphes (pensée et matière sonore) et en délimiterait arbitrairement les éléments. Cette fantasmagorie sur l'origine (considération de la pensée et de la substance sonore avant le langage) a été fermement critiquée par Hjelmslev : « la substance dépend exclusivement de la forme et [...] on ne peut en aucun cas lui prêter une existence indépendante » ([1943] 1968, p. 74). La même critique vaut contre ceux (Deleuze, Derrida, Milner, notamment) qui placent les différences à l'origine des éléments, réclamant pour ce faire la construction d'une «nouvelle logique ». Si les éléments n'ont qu'une définition différentielle (comme le montrent les tests de commutation et de permutation, $c f$. Hjelmslev ibid. sq.), si leur réalité ultime est leur valeur, il n'en demeure pas moins qu'il faut des éléments pour qu'il y ait des différences entre eux. Ce que propose Hjelmslev, ce n'est pas une nouvelle logique mais l'impossibilité de considérer de façon indépendante éléments et relations.

Nous pouvons construire des "faits de langue ", par exemple, un système phonologique ou un système verbal. C'est ce que font les grammairiens depuis

La différence de valeur entre sheep et mouton tient à ce que le premier a à côté de lui un second terme, ce qui n'est pas le cas pour le mot français » (Cours..., p. 160). Cela n'implique pas que Grasserie soit la source de Saussure, mais témoigne plutôt de la grande circulation intellectuelle du thème et des exemples

12 Les entrées des dictionnaires de synonymes se présentent sous la forme d'une liste des mots synonymes (donc comprennent au moins deux items) ; la série que cite le texte de Saussure figure dans la plupart des dictionnaires.

13 « (...) la définition de la partie présuppose celle de la classe », Hjelmslev [1943] 1968, p. 48. 
l'Antiquité. Mais cela n'implique pas qu'il existe une « langue » dans l'épaisseur du monde, parmi les objets naturels, quelque chose qui soit " un système où tout se tient ». Même dans les constructions des grammairiens, il y a parfois des inconsistances. Le français a perdu le système casuel du latin, mais il a conservé un système casuel pour les pronoms aussi bien personnels que relatifs ${ }^{14}$.

La réalité que mettent au jour la commutation ou la permutation dépend évidemment de la taille et de la nature du corpus considéré. Comme on ne peut tout envisager empiriquement (en particulier ce qui n'a pas eu lieu) l'existence de la langue est avant tout une projection ou un axiome (Auroux 1998, p. 98). Elle se heurte à la variation dialectale que Meillet dès 1908, plaçait à l'origine des « langues communes » et à ses limites. L'école de Prague considérait que les dialectes étaient bien des dialectes de la même langue lorsqu'ils connaissaient des évolutions parallèles. Pour Troubetzkoy, dans un travail perdu des années 1920, qu'il résume dans ses Notes autobiographiques ${ }^{15}$, le " slave commun " est un ensemble de dialectes qui ont connu des innovations phonétiques et morphologiques propagées dans toutes les langues slaves et dont l'évolution couvre deux millénaires et demi.

On peut dire que l'axiome de la langue repose sur un certain nombre d'hypothèses que l'on peut considérer comme grossièrement équivalentes. La première est celle de Saussure. Elle est exprimée dans l'idée que la langue existe sous forme d'empreintes déposées dans le cerveau des individus, à peu près comme un dictionnaire dont tous les exemplaires identiques seraient répartis entre les individus (Cours..., éd. Engler, fasc. 1, p. 57). La seconde est celle de Chomsky (passim) assertant l'existence d'un locuteur-auditeur idéal en lieu et place des sujets linguistiques (Auroux 1998, p. 269 ; Muni Toke 2013). La troisième est celle de Milner, qui la nomme « isotopie » : de quelque point de vue qu'on la prenne une langue demeure identique à elle-même en tous ses points (1978, p. 20). On peut remarquer que comme élément du réel une telle langue est « introuvable » (Gadet \& Pêcheux 1980). Les deux dernières positions sont plus radicales que la première, que Saussure tenait pour compatible avec l'idée, empruntée aux romanistes français, selon laquelle c'est le point de vue du linguiste qui créé la langue.

L'axiome de la langue fait de celle-ci une totalité uniforme et atemporelle. Nous avons souvent critiqué, comme certains chercheurs (Harris 1980, 1981 ; Harris [ed.] 2001 ; Calvet 2004), le « mythe de la langue » (Auroux 1998, 2010). Une telle unité correspond à l'imaginaire des locuteurs et aux constructions techniques des grammairiens et lexicologues, dont les outils linguistiques (Auroux 1994) aident les différentes politiques linguistiques et sociales à forger quelque apparence de cette unité. Leur réussite est parfois éclatante. Mais une

14 La présence de sous-systèmes relativement indépendants rend généralement l'ensemble plus stable (Simon 1991).

15 Publiées en tête de la traduction française des Principes de Phonologie. 
telle langue se heurte, du coup, à la diversité des actes de paroles (la performance) dont chacun admet la créativité. On a fait, parfois, du dualisme langue/parole ou compétence/performance une aporie insoluble. Pour la résoudre, il suffit d'abandonner le réalisme de la « langue » et de reconnaître qu'il s'agit d'une construction.

L'activité langagière quotidienne des hommes relève de l'action. La réversibilité du locuteur et du récepteur - l'isotopie de la langue - est inconcevable. Si nous avions la même chose dans notre tête ${ }^{16}$ que notre interlocuteur la compréhension serait tout simplement impossible. Il n'y a compréhension que sous forme de différences, de manque et d'un fond irréductible d'incompréhension, pas dans la répétition du même. C'est l'irréversibilité et la différence qui permettent la compréhension (et l'histoire). Celle-ci est du ressort de la théorie des jeux de coopération (Jaeger 2008). La variété est nécessairement première ${ }^{17}$. Il n'y a que des individus qui communiquent avec différentes compétences au sein de leur espace de vie structuré par leur présence à tous, leurs institutions, leurs outils (dont leurs outils linguistiques, intellectuels et culturels) le monde qu'ils habitent, ce qu'on peut appeler une « hyperlangue » (Auroux 1998, p. 113 sq.).

\section{RÉFÉRENCES}

Albano Leoni, Federico, 2009. Dei suoni e dei sensi. Il volto fonico della parole, Bologne, Il Mulino [traduction française 2014, Lyon, ENS Éditions].

Assoun, Paul-Laurent, 2009. Lacan, Paris, PUF.

Auroux, Sylvain, 1984. " D’Alembert et les synonymistes ", Dix-huitième Siècle, 16, 93108.

- 1985. «Deux hypothèses sur les sources de la conception saussurienne de la valeur linguistique », Travaux de linguistique et de littérature, B-1, 188-191.

- 1986. «Les synonymistes et la contrainte de scientificité : Roubaud 1785 », Autour de Féraud, la lexicographie en France de 1762 à 1885, Collection de l'ENS JF, 29, 73-81.

- 1993. La logique des idées, Montréal/Bellarmin, Paris/Vrin.

- 1994. "L'hypothèse de l'histoire et la sous-détermination grammaticale ", Langages, $118,25-40$.

- 1998. Le langage, la raison et les normes, Paris, PUF.

16 C'est l'un des traits constants de la philosophie occidentale de présupposer que l'humanité existe sous forme de sujets aux capacités et contenus cognitifs, rigoureusement identiques. Peut-on imaginer un seul instant que nous ayons dans notre tête les différentes rubriques d'un dictionnaire ? Si nos compétences étaient identiques à quoi servirait un dictionnaire monolingue ? Si tout était dans notre tête il faudrait que nous ayons connaissance de toutes les ambiguités possibles d'un mot. Pourquoi aurait-il fallu aux Grecs l'étude des Réfutations Sophistiques d'Aristote?

17 Cette question est évidemment cruciale pour la linguistique (« (...) the apparent richness and diversity of linguistic phenomena is illusory and epiphenomenal, the result of interaction of fixed principles under slightly varying conditions ", Chomsky 1995, p. 8). Mais il est clair qu'elle possède une profondeur éthique et politique qui va largement au-delà de cette discipline. 
- 2007. La question de l'origine des langues, suivi de L'historicité des sciences, Paris, PUF.

- 2008. « Brève histoire de la proposition », Cahiers de l'ILSL, 25, 15-34.

- 2009. La philosophie du langage, Paris, PUF.

- 2010. "Sur le mythe de la langue », in Gasquet-Cyrus, Médéric, Giacomi, Alain, Touchard, Yvonne, Véronique, Daniel (éd.), Pour la (socio)linguistique. Pour LouisJean-Calvet, Paris, L'Harmattan, 173-178.

- à paraître. Le structuralisme, Paris, PUF.

Auroux, Sylvain, Deschamps, Jacques, Kouloughli, Djamel, 1996. La Philosophie du langage, Paris, PUF.

Baker, Mark C., 1988. Incorporation, Chicago, University of Chicago Press.

Berlin, Brent \& Kay, Paul, 1969. Basic Color Terms: Their Universality and Evolution, Berkeley, University of California Press.

Bertrand, Elie, 1758. Recherches sur les langues modernes et anciennes de la Suisse et principalement du Canton de Vaud, Genève, Philibert.

Calvet, Louis-Jean, 2004. Essais de linguistique. La langue est-elle une invention des linguistes?, Paris, Plon.

Cardona, Giorgio Raimondo, 1985. La foresta di piume. Manuale di etnoscienza, Roma/ Bari, Laterza.

- 1990. I Linguaggi del Sapere, Roma/Bari, Laterza.

Chomsky, Noam, [1957] 1969. Structures syntaxiques, Paris, Seuil.

- 1990. " On Formalisation and Formal Linguistics ", Natural Language and Linguistic Theory, 8, 143-147.

- 1995. The minimalist Program, Cambridge, The MIT Press.

Cohen, Marcel, 1972. Matériaux pour une sociologie du langage, t. II, Paris, François Maspero.

Colombat, Bernard (dir.), 1988. Les parties du discours, Paris, Larousse, coll. « Langages » 92.

Daladier, Anne, 1990. « Aspects constructifs des grammaires de Harris », Paris, Larousse, coll. « Langages » 49, 57-84.

Denys le Thrace, 1989, La technê grammatikê, trad., introd. et notes par J. Lallot, Paris, CNRS Éditions.

Dubois, Danièle \& Cance, Caroline, 2009. « Mettre un terme aux couleurs de base : déconstruction d'un paradigme dominant ", in Dubois, Danièle (dir.), Le sentir et le dire, Paris, L'Harmatan, 75-104.

Durkheim, Émile, [1937] 1977. Les règles de la méthode sociologique, Paris, PUF.

Fodor, Jerry A., 1983. The Modularity of Mind, Cambridge, The MIT Press.

Gadet, Françoise \& Pêcheux, Michel, 1981. La langue introuvable, Paris, François Maspero.

Gauger, Hans-Martin, 1973. Die Anfänge der Synonymik. Girard (1718) und Roubaud (1785) ; ein Beitrag zur Geschichte der lexikalischen Semantik, Tübingen, G. Narr, « Tübinger Beiträge zur Linguistik » 39.

Goodman, Nelson, 1983. Facts, Fiction and Forecast, Harvard University Press [1ère éd. plus courte en 1954].

Grasserie, Raoul de la, 1908. Essai d'une sémantique intégrale, vol. 2, Paris, Leroux.

Harris, Roy, 1980. The language makers, London, Duckworth.

- 1981. The language myth, London, Duckworth.

- (ed.), 2001. The language myth in western culture, Richmond, Curzon Press.

Hjelmslev, Louis, [1943] 1968. Prolégomènes à une théorie du langage, traduction française, Paris, Minuit.

Fournier, Jean-Marie (dir.), 2007. L'exemple, Paris, Larousse/Armand Colin, coll. « Langages » 166.

Hockett, Charles F., 1954. " Two models of grammatical description », Word, 10, 210-234.

Humboldt, Wilhelm Von, [1836] 1974. Introduction à l'ouvre sur le kavi et autres essais, traduction française de Pierre Caussat, Paris, Le Seuil. 
Jaeger, Gerhard, 2008. "Applications of Game Theory in Linguistics », Language and Linguistics Compax, 406-421.

Katz, Jerrold J., 1981. Language and other Abstracts Objects, Totawa N.J., Rowman and Littelfield.

Lacan, Jacques, 1966. « La science et la vérité », Écrits, Paris, Le Seuil, 855-877.

Launey, Michel, 1994. Une grammaire omni-prédicative. Essai sur la morphosyntaxe du nahuatl classique, Paris, CNRS Éditions.

Le Bon, Gustave, [1895] 1975. Psychologie des foules, Paris, CEPL.

Lemaréchal, Alain, 1989. Les parties du discours. Sémantique et syntaxe, Paris, PUF.

Lerat, Pierre, 1995. Les langues spécialisées, Paris, PUF.

Macpherson, Crawford Brough, [1962] 1971. La théorie politique de l'individualisme possessif, traduction française, Paris, Gallimard.

Lévi-Strauss, Claude, 1950. «Introduction à l'œuvre de Marcel Mauss », in Mauss, Marcel, Sociologie et anthropologie, Paris, PUF.

Milner, Jean-Claude, 1978. L'amour de la langue, Paris, Seuil [voir notamment «5. Détour par les chicanes du Tout », 70-84].

Meillet, Antoine, 1908. Les dialectes indoeuropéens, Champion, Paris.

Muni Toke, Valelia, 2013. "Le locuteur natif et son idéalisation : un demi-siècle de critiques », Histoire, Épistémologie, Langage, XXXV-2, 5-15.

Parkinson, Franck, 1972. " Linguistic and Mathematical Infinity », $C F F, 27,55-64$.

Quine Willard von Orman, 1960. Word and Object, Cambridge (Ma), The MIT Press.

Rickey, V.F., 1975. « Creative definitions in propositionnel calcul », Notre Dame Journal of Formal Logic, 16-2, 283-294.

Saussure, Ferdinand de ([1916] 1967-1974). Cours de linguistique générale, éd. critique par R. Engler, Wiesbaden, Otto Harrassowitz.

Simon, Herbert, 1991. Sciences des systèmes, science de l'artificiel, t.f., Paris, Dunod

Singh, Rajendra \& Ford Alan, 1980. "Flexion, Derivation and Panini », in Koerner, E. F. Konrad (ed.), Progress in Linguistic Historiography, Amsterdam, John Benjamins, 323-332.

Tarski, Alfred, [1933] 1972. "Le concept de vérité dans les langages formalisés », in Tarski, Alfred, Logique, sémantique, métamathématique, Paris, Armand Colin, I, 159-269.

Troubetzkoy, Nicolas S., ([1939] 1947). Principes de Phonologie, trad. fr. par J. Cantineau, Paris, Klincksieck.

Zuber, R., 1990. "Définition (- créative) », in Auroux, Sylvain (dir.), Les Notions philosophiques. Dictionnaire, Paris, PUF, I, 568. 